\title{
$\sigma$-HEREDITARILY CLOSURE-PRESERVING $k$-NETWORKS AND $g$-METRIZABILITY
}

\author{
YOSHIO TANAKA \\ (Communicated by Dennis Burke) \\ Dedicated to Professor Ryosuke Nakagawa on his sixtieth birthday
}

\begin{abstract}
We show that a regular space is $g$-metrizable if and only if it is a weakly first countable space with a $\sigma$-hereditarily closure-preserving $k$-network.
\end{abstract}

\section{INTRODUCTION}

Let $X$ be a space. For each $x \in X$, let $T_{x}$ be a collection of subsets of $X$ such that any element of $T_{x}$ contains $x$. Following Arhangel'skii [1], the collection $T_{c}=\bigcup\left\{T_{x} ; x \in X\right\}$ is a weak base for $X$ if (a) and (b) below are satisfied (see [4] or [8]).

(a) For each $B_{1}, B_{2} \in T_{x}$, there exists $B_{3} \in T_{x}$ such that $B_{3} \subset B_{1} \cap B_{2}$.

(b) $U \subset X$ is open if and only if, for each $x \in U$, there exists $B \in T_{x}$ such that $B \subset U$.

We call the $T_{x}$ a weak neighborhood base for $x$ in $X$, and each element of $T_{x}$ a weak neighborhood of $x$. A space $X$ is weakly first countable $(=g$-first countable [15], or $X$ satisfies the weak first axiom of countability [1]) if $X$ has a weak base $T_{c}$ such that each $T_{x}$ is countable. Every first countable space, or symmetric space (in the sense of [1, p. 125]) is weakly first countable. Every weakly first countable $\sigma$-space is a symmetric space (cf. [1]).

We recall that a space $X$ is sequential (resp. a $k$-space) if $F \subset X$ is closed in $X$ if and only if $F \cap C$ is closed in $C$ for every convergent sequence $C$ containing its limit point (resp. a compact subset $C$ ) in $X$. Every weakly first countable space is sequential (for example, [15]), and every sequential space is a $k$-space.

F. Siwiec [15] introduced the notion of $g$-metrizable spaces as a generalization of metric spaces. A space $X$ is $g$-metrizable if it has a $\sigma$-locally finite weak base. A space $X$ is metrizable if and only if it is a Fréchet, $g$-metrizable space

Received by the editors November 13, 1989 and, in revised form, April 3, 1990.

1980 Mathematics Subject Classification (1985 Revision). Primary 54E99.

Key words and phrases. Hereditarily closure-preserving collection, $k$-network, weak base, $g$ metrizable space. 
[15]. Here a space $X$ is Fréchet if, whenever $x \in \bar{A}$, there exists a sequence in $A$ converging to the point $x$. Other interesting properties of $g$-metrizable spaces are obtained by N. Jakovlev [8] (see also [18]).

Let $\mathscr{A}=\left\{A_{\alpha} ; \alpha \in A\right\}$ be a collection of subsets of a space $X$. We recall that $\mathscr{A}$ is closure-preserving if $\overline{\bigcup\left\{A_{\alpha} ; \alpha \in B\right\}}=\bigcup\left\{\bar{A}_{\alpha} ; \alpha \in B\right\}$ for any $B \subset A$. $\mathscr{A}$ is hereditarily closure-preserving if $\bigcup\left\{B_{\alpha} ; \alpha \in B\right\}=\bigcup\left\{\bar{B}_{\alpha} ; \alpha \in B\right\}$ whenever $B \subset A$ and $B_{\alpha} \subset A_{\alpha}$ for each $\alpha \in B$. A $\sigma$-hereditarily closure-preserving collection is the union of countably many hereditarily closure-preserving collections. We shall use " $\sigma$-CP" (resp. " $\sigma$-HCP") instead of " $\sigma$-closure preserving" (resp. " $\sigma$-hereditarily closure-preserving") in this paper. Obviously, every $\sigma$-locally finite collection is $\sigma$-HCP.

Let $\mathscr{C}$ be a cover of a space $X$. We recall that $\mathscr{C}$ is network (= net) for $X$ if for each $x \in X$ and neighborhood $U$ of $x$, there exists $C \in \mathscr{C}$ such that $x \in C \subset U . \mathscr{C}$ is a $k$-network if whenever $K \subset U$ with $K$ compact and $U$ open in $X$, then $K \subset \cup \mathscr{C}^{\prime} \subset U$ for some finite $\mathscr{C}^{\prime} \subset \mathscr{C}$. A space is an $\aleph$-space [14] if it has a $\sigma$-locally finite $k$-network. Every $\sigma$-HCP weak base is a $k$-network [4].

L. Foged [4] proved that a space is $g$-metrizable if and only if it is a weakly first countable space with a $\sigma$-locally finite $k$-network. (Hence, a $g$-metrizable space is equal to a symmetric, $\aleph$-space.)

In view of Foged's result, Y. Kotake [11] asked whether every weakly first countable space with a $\sigma$-HCP $k$-network is $g$-metrizable. Related to this question, he also posed the following problem in [12]: Characterize a space having a $\sigma$-HCP weak base.

In this paper, we give an affirmative answer to the first question and, as for the second question, we show that every $k$-space with a $\sigma$-HCP weak base is precisely a $g$-metrizable space.

We assume that all spaces are regular, and all maps are continuous and onto.

\section{RESULTS}

For a cover $\mathscr{C}$ of a space $X$, we consider the following condition $(*)$, which is labeled $\left(C_{2}\right)$ in [17].

(*) If $A$ is a sequence converging to a point $x$ and $U$ is a neighborhood, then there exists $C \in \mathscr{C}$ such that $C \subset U$ and $C$ contains a subsequence of A.

Obviously, every $k$-network satisfies $(*)$.

Lemma 1 ([17]). Every $\sigma$-HCP cover satisfying (*) is a k-network.

Lemma 2. Let $X$ be a $k$-space, or a Lindelöf space. If $X$ has a $\sigma$-HCP weak base, then it is weakly first countable.

Proof. Let $\mathscr{B}=\bigcup\left\{\mathscr{B}_{n} ; n \in N\right\}$ be a $\sigma$-HCP weak base for $X$. Let $X$ be a $k$-space. Since $X$ has a $\sigma$-CP network of closed subsets, each point of $X$ is a $G_{\delta}$-set. Then any compact subset $K$ of $X$ is first countable, for each point of $K$ is a $G_{\delta}$-set in $K$. Then it is easy to show that a $k$-space $X$ is sequential. 
Let $x \in X$ be not isolated. Then there exists a sequence $A$ converging to $x$ with $A \not \supset x$. Note that any weak neighborhood of $x$ contains $A$ eventually, because $A \cup\{x\}$ is closed, but $A$ is not closed in $X$, but each $\mathscr{B}_{n}$ is HCP and any infinite subset of $A$ is not closed in $X$. Then for any $n \in N, T_{x} \cap \mathscr{B}_{n}$ is finite. Hence $T_{x} \cap \mathscr{B}$ is countable. Then $X$ is weakly first countable. Next, let $X$ be Lindelöf. Then for any nonisolated point $x$ in $X$ and any $n \in N$, $T_{x} \cap \mathscr{B}_{n}$ is countable. Indeed, suppose that $T_{x} \cap \mathscr{B}_{n}$ is not countable for some $n \in N$. Then there exists a subcollection $\left\{B_{\alpha} ; \alpha<\omega_{1}\right\}$ of $T_{x} \cap \mathscr{B}_{n}$. Since $x$ is not isolated in $X$, for any $B_{\alpha}$ and any neighborhood $G$ of $x$, $B_{\alpha} \cap G \cap(X-\{x\}) \neq \varnothing$. Then for each $\alpha<\omega_{1}$, there exists $x_{\alpha} \in B_{\alpha} \cap$ $\left(X-\left\{x_{\beta} ; \beta<\alpha\right\}\right) \cap(X-\{x\})$. Then $\left\{x_{\alpha} ; \alpha<\omega_{1}\right\}$ is a discrete, closed subset of $X$ with cardinality $\omega_{1}$. But $X$ is Lindelöf. This is a contradiction. Then for any $n \in N, T_{x} \cap \mathscr{B}_{n}$ is countable. Hence $X$ is also weakly first countable.

Lemma 3 ([4]). Every weakly first countable space with a $\sigma$-locally finite $k$ network is g-metrizable.

Lemma 4. Let $X$ have a $\sigma$-CP $k$-network. Then, for each closed subset $F$ of $X$, there exists a sequence $\left\{V_{n} ; n \in N\right\}$ of open subsets of $X$ such that each $V_{n}$ contains $F$, and for each sequence $\left\{x_{n} ; n \in N\right\}$ converging to a point $x \notin F$, some $V_{n}$ contains only finitely many $x_{n}$, but does not contain $x$.

Proof. Let $\mathscr{C}=\bigcup\left\{\mathscr{C}_{n} ; n \in N\right\}$ be a $\sigma$-CP $k$-network for $X$ by closed subsets of $X$ such that $\mathscr{C}_{n} \subset \mathscr{C}_{n+1}$ for each $n \in N$. For each $n \in N$, let $V_{n}=$ $X-\bigcup\left\{C \in \mathscr{C}_{n} ; C \cap F=\varnothing\right\}$. Then each $V_{n}$ contains $F$. Since $\mathscr{C}$ is a $k$-network with $\mathscr{C}_{n} \subset \mathscr{C}_{n+1}$, there exist $m, n \in N$ and a finite union $A$ of elements of $\mathscr{C}_{n}$ such that $\left\{x_{i} ; i \geq m\right\} \cup\{x\} \subset A \subset X-F$. Then $V_{n}$ contains only finitely many $x_{n}$, and does not contain $x$.

Lemma 5. Let $X$ have a weak base $\bigcup\left\{T_{x} ; x \in X\right\}$ such that each $T_{x}$ is countable. Let $D$ be a closed discrete subset of $X$. If $X$ has a $\sigma-\mathrm{HCP} k$-network, then there exists a $\sigma$-discrete collection $\mathscr{D}$ in $X$ such that, for each $d \in D, \mathscr{D} \cap T_{d}$ is a weak base for $d$ in $X$.

Proof. First we note that, among regular spaces, if $\mathscr{A}$ is HCP, then so is $\{\bar{A} ; A \in \mathscr{A}\}$. Thus, $X$ admits a $\sigma$-HCP $k$-network $\mathscr{C}=\bigcup\left\{\mathscr{C}_{n} ; n \in N\right\}$ of closed subsets such that $\mathscr{C}_{n} \subset \mathscr{C}_{n+1}$ for each $n \in N$. Let $D=\left\{x_{\alpha} ; \alpha \in A\right\}$. For each $n \in N$ and each $\alpha \in A$, let

$$
\mathscr{C}_{n, \alpha}=\left\{C \in \mathscr{C}_{n} ; C \ni x_{\alpha} \text { and } C \cap\left\{x_{\beta} ; \beta \neq \alpha\right\}=\varnothing\right\} \text {, }
$$

let

and let

$$
C(n, \alpha)=\bigcup \mathscr{C}_{n, \alpha}
$$

$$
C^{*}(n, \alpha)=C(n, \alpha)-\bigcup\{C(n, \beta) ; \beta \neq \alpha\} .
$$

For each $d \in D$, we can assume that $T_{d}=\left\{Q_{n}(d) ; n \in N\right\}$ is a decreasing weak neighborhood base for $d$ in $X$ consisting of closed subsets of $X$. For 
each $x_{\alpha} \in D$, there exist $m, n \in N$ such that $Q_{n}\left(x_{\alpha}\right) \subset C^{*}(m, \alpha)$. Indeed, there exists $m \in N$ such that $Q_{m}\left(x_{\alpha}\right) \subset C(m, \alpha)$. To show this, suppose that $Q_{n}\left(x_{\alpha}\right) \not \subset C(n, \alpha)$ for any $n \in N$. Then there exists a sequence $A=\left\{a_{n} ; n \in\right.$ $N\}$ such that $a_{n} \in Q_{n}\left(x_{\alpha}\right)-C(n, \alpha)$. Since the sequence $A$ converges to $x_{\alpha}$, and $\mathscr{C}$ is a $k$-network of closed subsets, there exists $C \in \mathscr{C}_{n}$ for some $n \in N$ such that $C$ contains $x_{\alpha}$ and a subsequence of $A$, and $C \cap\left\{x_{\beta} ; \beta \neq \alpha\right\}=\varnothing$. Then $C \subset C(m, \alpha)$ for $m \geq n$. Hence there exists $a_{k} \in C(k, \alpha)$ for some $k \geq n$. This is a contradiction. Then $Q_{m}\left(x_{\alpha}\right) \subset C(m, \alpha)$ for some $m \in N$. On the other hand, since $x_{\alpha} \notin \bigcup\{C(m, \beta) ; \beta \neq \alpha\}$, there exists $i \in N$ such that $Q_{i}\left(x_{\alpha}\right) \cap \bigcup\{C(m, \beta) ; \beta \neq \alpha\}=\varnothing$. Let $n \geq m, i$. Then $Q_{n}\left(x_{\alpha}\right) \subset C^{*}(m, \alpha)$. Now, for each $m, n \in N$, let $\mathscr{D}_{m n}=\left\{Q_{n}\left(x_{\alpha}\right) ; Q_{n}\left(x_{\alpha}\right) \subset C^{*}(m, \alpha), \alpha \in A\right\}$. For each $m \in N$, let $\mathscr{D}_{m}=\bigcup\left\{\mathscr{D}_{m n} ; n \in N\right\}$, and let $D_{m}=\bigcup \mathscr{D}_{m}$. Then $D \subset$ $\bigcup\left\{D_{m} ; m \in N\right\}$ by the above arguments. We note that for each $m \in N$ and $\alpha$, $\beta \in A$, if $\alpha \neq \beta$, then $C^{*}(m, \alpha) \cap C^{*}(m, \beta)=\varnothing$, and $\mathscr{C}_{m, \alpha} \cap \mathscr{C}_{m, \beta}=\varnothing$. But for each $Q_{n}\left(x_{\alpha}\right) \in \mathscr{D}_{m n}, Q_{n}\left(x_{\alpha}\right) \subset C^{*}(m, \alpha)$ and $Q_{n}\left(x_{\alpha}\right) \subset C(m, \alpha)$. Then, since each $\mathscr{C}_{m}$ is HCP, each $\mathscr{D}_{m n}$ is a disjoint, CP collection of closed subsets of $X$; hence, it is discrete in $X$. Then each $\mathscr{D}_{m}$ is a $\sigma$-discrete collection in $X$. Let $\mathscr{D}=\bigcup\left\{\mathscr{D}_{m} ; m \in N\right\}$. Hence $\mathscr{D}$ is a $\sigma$-discrete collection in $X$ such that, for each $d \in D, \mathscr{D} \cap T_{d}$ is a weak base for $d$ in $X$.

Now, we prove the main theorem.

Theorem 6. The following are equivalent:

(a) $X$ is $g$-metrizable.

(b) $X$ is a $k$-space with a $\sigma$-HCP weak base.

(c) $X$ is a weakly first countable space with a $\sigma$-HCP $k$-network.

Proof. Every $g$-metrizable space is sequential [15]; hence, it is a $k$-space. Then (a) $\Rightarrow$ (b) holds. We note that, for any sequence converging to a point $x$, any weak neighborhood of $x$ contains the sequence eventually. Then $(b) \Rightarrow$ (c) follows from Lemmas 1 and 2. We prove (c) $\Rightarrow$ (a). By Lemmas 1 and 3 , it suffices to show that $X$ has a $\sigma$-locally finite cover satisfying $(*)$. Let $\mathscr{C}=\bigcup\left\{\mathscr{C}_{n} ; n \in N\right\}$ be a $\sigma$-HCP $k$-network for $X$ by closed subsets of $X$ such that $\mathscr{C}_{n} \subset \mathscr{C}_{n+1}$ for each $n \in N$. For each $n \in N$, let $D_{n}=\left\{x \in X ; \mathscr{C}_{n}\right.$ is not point-finite at $x\}$. Since $X$ is weakly first countable, it is sequential. Then, since each $\mathscr{C}_{n}$ is HCP, it follows that each $D_{n}$ is closed, and discrete in $X$. By Lemma 4, for each $n \in N$, there exists a collection $\left\{V_{m n} ; m \in N\right\}$ of open subsets of $X$ satisfying the condition in Lemma 4 with respect to the closed subset $D_{n}$. For each $m, n \in N$, let $\mathscr{C}_{m n}=\left\{C-V_{m n} ; C \in \mathscr{C}_{n}\right\}$. Let $\mathscr{C}^{\prime}=\bigcup\left\{\mathscr{C}_{m n} ; m, n \in N\right\}$. Since each $\mathscr{C}_{m n}$ is a point-finite and CP collection of closed subsets of $X$, it is locally finite in $X$. Hence $\mathscr{C}^{\prime}$ is a $\sigma$-locally finite collection of closed subsets of $X$. Let $\bigcup\left\{T_{x} ; x \in X\right\}$ be a weak base for $X$ such that each $T_{x}$ is countable. Since each $D_{n}$ is a closed discrete subset of $X$, by Lemma 5 there exists a $\sigma$-discrete collection $\mathscr{D}_{n}$ in $X$ such that, for each $d \in D_{n}, \mathscr{D}_{n} \cap T_{d}$ is a weak base for $d$ in $X$. Let $\mathscr{D}=\bigcup\left\{\mathscr{D}_{n} ; n \in N\right\}$. 
Let $\mathscr{P}=\mathscr{C}^{\prime} \cup \mathscr{D}$. Then $\mathscr{P}$ is a $\sigma$-locally finite cover of $X$. We show that $\mathscr{P}$ satisfies $(*)$. Let $U$ be open in $X$ and $A$ be a sequence converging to a point $x$ in $U$. Let $S=X-\bigcup\left\{D_{n} ; n \in N\right\}$. If $x \in S$, then there exists an element $C$ of $\mathscr{C}^{\prime}$ such that $C \subset U$ and $C$ contains a subsequence of $A$. If $x \notin S$, then there exists an element $D$ of $\mathscr{D}$ such that $D \subset U$ and $D$ contains $A$ eventually. Then $\mathscr{P}$ satisfies $(*)$. Hence $X$ has a $\sigma$-locally finite cover $\mathscr{P}$ satisfying $(*)$.

Lemma 7 ([15]). Every Fréchet, g-metrizable space is metrizable.

Remark 8. (1) As for networks (= nets), the following (a) holds by [16]: (a) $X$ has a $\sigma$-HCP network $\Leftrightarrow X$ has a $\sigma$-CP network $\Leftrightarrow X$ has a $\sigma$-discrete network ( $\Leftrightarrow X$ is a $\sigma$-space). In (a), we cannot replace "network" by " $k$-network." Indeed, not every space with a $\sigma$-HCP $k$-network has a $\sigma$-locally finite $k$ network; see Theorem 14. However, among weakly first countable spaces, we have the following (b) in view of [8, Theorem 5] (or the proof of [4, Theorem 2.4]) and Theorem 6: (b) Let $X$ be weakly first countable. Then $X$ has a $\sigma$-HCP $k$-network $\Leftrightarrow X$ has a $\sigma$-discrete $k$-network $\Leftrightarrow X$ has a $\sigma$-discrete weak base $(\Leftrightarrow X$ is a $g$-metrizable space).

(2) In Theorem 6 , we cannot weaken " $\sigma$-HCP $k$-network" (resp. " $\sigma$-HCP weak base") to " $\sigma$-CP $k$-network" (resp. " $\sigma$-CP weak base"), by (a) and (b) below: (a) There exists a separable, first countable space $X$ with a $\sigma$-CP open base, but $X$ is not $g$-metrizable; (b) There exists a weakly first countable, CW-complex $X$, but $X$ is not $g$-metrizable. As for (a), the nonmetrizable, separable, first countable $M_{1}$-space $X$ in [3, Example 9.2] is the desired space by Lemma 7 .

As for (b), let $X$ be the CW-complex in [17, p. 336] such that $X$ is the quotient, finite-to-one image of a metric space, but $X$ has no $\sigma$-HCP $k$-networks. Note that every $\mathrm{CW}$-complex has a $\sigma-\mathrm{CP}$ open base by [3, Corollary 8.6], and that every quotient, finite-to-one image of a metric space is weakly first countable in view of $[1$, p. 125]. Then $X$ is the desired space.

In the following corollary, (a) $\Leftrightarrow(b)$ is due to [2], and (a) $\Leftrightarrow(\mathbf{c})$ is Guthrie's unpublished result.

Corollary 9. The following are equivalent:

(a) $X$ is metrizable.

(b) $X$ has a $\sigma-\mathrm{HCP}$ open base.

(c) $X$ is a first countable space with a $\sigma$-HCP $k$-network.

Proof. As for (b), note that every space with a $\sigma$-HCP open base is first countable by [2, Lemma 4]. Then the corollary follows from Theorem 6 and Lemma 7.

A space is g-second countable [15] if it has a countable weak base. The following Corollary is obtained from Theorem 6 and Lemma 2. 
Corollary 10. The following are equivalent:

(a) $X$ is a $g$-second countable space.

(b) $X$ is a Lindelöf space with a $\sigma$-HCP weak base.

(c) $X$ is a Lindelöf, weakly first countable space with a $\sigma$-HCP $k$-network.

The author has the following question in view of Theorem 6 and Corollary 10.

Question. Is every space with a $\sigma$-HCP weak base $g$-metrizable?

Let us give some partial answers to this question. We recall that a space $X$ is a $c$-space (or $X$ has countable tightness) if, whenever $x \in \bar{A}$, then $x \in \bar{C}$ for some countable $C \subset A$. Every $g$-metrizable (more generally, every sequential) space is a $c$-space; see [13, p. 123], for example. A space is meta-Lindelöf if every open cover has a point-countable open refinement.

Proposition 11. Let $X$ have a $\sigma$-HCP weak base. Then (a) or (b) below implies that $X$ is g-metrizable.

(a) $(\mathrm{CH}) . X$ is a separable space, or a c-space.

(b) $X$ is a meta-Lindelöf c-space.

Proof. For case (a), let $X$ be a separable space. Then, as is well known, $X$ has an open base of cardinality $\leq 2^{\omega}$, hence of cardinality $\leq \omega_{1}$. Let $\mathscr{B}=$ $\bigcup\left\{\mathscr{B}_{n} ; n \in N\right\}$ be a $\sigma$-HCP weak base for $X$. Let $x \in X$ be not isolated in $X$. Then for any $n \in N$ and for any $A \in T_{x} \cap \mathscr{B}_{n}, x$ is not isolated in $A$. Hence in view of [2, Corollary 2], $T_{x} \cap \mathscr{B}_{n}$ is countable for each $n \in N$. Then $T_{x} \cap \mathscr{B}$ is countable. Thus $X$ is weakly first countable, hence a $k$-space. Next, let $X$ be a $c$-space. Then it is easy to show that $F \subset X$ is closed in $X$ if $F \cap S$ is closed for every separable closed subset $S$ of $X$. Let $S$ be any separable closed subset of $X$. Since $S$ is closed in $X$, it has a $\sigma$-HCP weak base. Since $S$ is separable, it is a $k$-space, as seen above. Thus any separable closed subset of $X$ is a $k$-space. To show that $X$ is a $k$-space, for $F \subset X$, let $F \cap C$ be closed for every compact subset $C$ of $X$. Let $S$ be any separable closed subset of $X$. Then $F \cap K$ is closed for every compact subset $K$ of $S$. Since $S$ is a $k$-space, $F \cap S$ is closed. Then $F$ is closed in $X$. Thus $X$ is a $k$-space. Then for case (a), $X$ is a $k$-space. Hence it is $g$-metrizable, by Theorem 6 .

For case (b), note that every separable closed subset of a meta-Lindelöf space $X$ is Lindelöf. But every Lindelöf closed subset of $X$ is a $k$-space by Lemma 2. Since $X$ is a $c$-space, $X$ is $g$-metrizable in view of the proof for case (a).

Lemma 12. Let $f: X \rightarrow Y$ be a closed map. If $X$ has a $\sigma$-HCP $k$-network, then so does $Y$.

Proof. Let $\mathscr{C}$ be a $\sigma$-HCP $k$-network. Then $f(\mathscr{C})$ is a $\sigma$-HCP cover of $Y$. Then, by Lemma 1 , it suffices to show that $f(\mathscr{C})$ satisfies $(*)$. To show this, let $A$ be a sequence converging to $y$ in $Y$, and let $U$ be a neighborhood of $y$. Since $f$ is a closed map such that each point of $X$ is a $G_{\delta}$-set, in view of 
[17, Lemma 1.6] there exists a convergent sequence $B$ in $X$ such that $f(B)$ is a subsequence of $A$. Thus there exists $C \in \mathscr{C}$ such that $f(C) \subset U$ and $f(C)$ contains a subsequence of $A$. Hence $f(\mathscr{C})$ satisfies $(*)$.

As is well known, every perfect image of a metric space is metrizable. However, not every perfect image of a countable, $g$-metrizable space is $g$-metrizable [18]. We recall that every closed image of a metric space is metrizable if and only if it is first countable. As for $g$-metrizability, we have the following analogue by Lemma 12 and Theorem 6:

Theorem 13. Let $f: X \rightarrow Y$ be a closed map such that $X$ is g-metrizable. Then $Y$ is $g$-metrizable if and only if it is weakly first countable.

Finally, among Fréchet spaces, let us give a survey of characterizations for nice properties by means of $k$-networks or weak bases. We recall that a map $f: X \rightarrow Y$ is an $s$-map if $f^{-1}(y)$ is separable for each $y \in Y$. For $M_{3}$-spaces, see [3].

Theorem 14. Let $X$ be a Fréchet space. Then we have the following:

(a) $X$ is a metric space $\Leftrightarrow X$ has a $\sigma$-locally finite weak base $\Leftrightarrow X$ has a $\sigma$-HCP weak base.

(b) $X$ is the closed s-image of a metric space $\Leftrightarrow X$ has a $\sigma$-locally finite $k$-network.

(c) $X$ is the closed image of a metric space $\Leftrightarrow X$ has a $\sigma$-HCP $k$-network.

(d) $X$ is an $M_{3}$-space $\Leftrightarrow X$ has a $\sigma$-CP k-network $\Leftrightarrow X$ has a $\sigma$-CP weak base.

Proof. (a) follows from Theorem 6 and Lemma 7. (This is also shown in [11]. Indeed, every Fréchet space with a $\sigma$-HCP weak base has a $\sigma$-HCP open base. Then $X$ is metrizable by Corollary 9.) (b) is due to [6], (c) is due to [5], and the equivalence in the first part of (d) is due to [10]. Let $X$ be an $M_{3}$-space. By [7] or [9], $X$ has a $\sigma-\mathrm{CP}$ quasi-base $\mathscr{B}$; that is, for $x \in X$ and a neighborhood $U$ of $x$, there exists $B \in \mathscr{B}$ such that $x \in$ int $B \subset B \subset U$. For $x \in X$, let $T_{x}=\{B \in \mathscr{B} ; x \in$ int $B\}$. Then $\bigcup\left\{T_{x} ; x \in X\right\}$ is a $\sigma$-CP weak base for $X$. Thus $X$ has a $\sigma-\mathrm{CP}$ weak base. Conversely, let $\mathscr{B}$ have a $\sigma$-CP weak base. Since $X$ is Fréchet, for any $x \in X$ and for any $B \in T_{x} \cap \mathscr{B}, x \in$ int $B$. Hence $\mathscr{B}$ is a $\sigma-\mathrm{CP}$ quasi-base for $X$. Hence $X$ is an $M_{3}$-space by [3, Theorem 2.2].

\section{REFERENCES}

1. A. V. Arhangel' skii, Mappings and spaces, Russian Math. Surveys 21 (1966), 115-162.

2. D. Burke, R. Engelking, and D. Lutzer, Hereditarily closure-preserving collections and metrizability, Proc. Amer. Math. Soc. 51 (1975), 483-488.

3. J. G. Ceder, Some generalizations of metric spaces, Pacific J. Math. 11 (1961), 105-125.

4. L. Foged, On g-metrizability, Pacific J. Math. 98 (1982), 327-332.

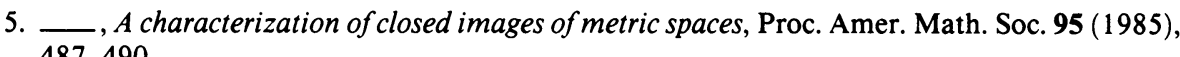
487-490. 
6. Z. Gao and Y. Hattori, A characterization of closed s-images of metric spaces, Questions Answers Gen. Topology 4 (1986/87), 147-151; Tsukuba J. Math. 2 (1987), 367-370.

7. G. Gruenhage, Stratifiable spaces are $M_{2}$, Topology Proc. 1 (1976), 221-226.

8. N. N. Jakovlev, On g-metrizable spaces, Soviet Math. Dokl. 17 (1976), 156-159.

9. H. Junnila, Neighbornets, Pacific J. Math. 76 (1978), 83-108.

10. Y. Kanatani, N. Sasaki, and J. Nagata, New characterizations of some generalized metric spaces, Math. Japon. 5 (1985), 805-820.

11. Y. Kotake, personal communication.

12. __ On g-metrizable spaces, RIMS Kokyuroku, Kyoto Univ. 584 (1986), 46-51.

13. E. Michael, A quintuple quotient quest, Gen. Topology Appl. 2 (1972), 91-138.

14. P. O'Meara, On paracompactness in function spaces with the compact-open topology, Proc. Amer. Math. Soc. 29 (1971), 183-189.

15. F. Siwiec, On defining a space by a weak base, Pacific J. Math. 52 (1974), 233-245.

16. F. Siwiec and J. Nagata, A note on nets and metrization, Proc. Japan Acad. 44 (1968), 623-627.

17. Y. Tanaka, Point-countable covers and k-networks, Topology Proc. 12 (1987), 327-349.

18. __ Symmetric spaces, g-developable spaces and g-metrizable spaces, Math. Japon. (to appear).

Department of Mathematics, Tokyo Gakugei University, Koganei-Shi, Tokyo (184), JAPAN 